\title{
"¿Hay aspectos de la reforma escolar mejicana que convenga hacer nuestros?" Pregunta planteada en una reunión de la Unión Nacional de Magisterio (U.N.M) de Uruguay en 1938
}

\author{
"Are there aspects of the Mexican school reform that we should \\ consider make our own?" Question posed at a meeting of the \\ National Union of Teachers (U.N.M) of Uruguay in 1938
}

\section{Pamela Reisin}

Orcid: https://orcid.org/0000-0003-2226-6240

CONICET, Universidad Nacional de Córdoba, Córdoba, Argentina

pame.reisin@gmail.com

DOI: 10.21680/2596-0113.2021v4n0ID25688

Citation: Reisin, P. (2021). “¿Hay aspectos de la reforma escolar mejicana que convenga hacer nuestros?"

Pregunta planteada en una reunión de la Unión Nacional de Magisterio (U.N.M) de Uruguay en 1938. History of

Education in Latin America - HistELA, 4, e25688.

Competing interests: The author have declared that no competing interests exist.

Editor: Olivia Morais de Medeiros Neta

Received: 23/06/2021

Approved: $30 / 07 / 2021$

\section{OPEN ACCESS}

\section{Resumen}

Se indagará en la pedagogía ruralista uruguaya y sus conexiones con la educación rural mexicana (1930-1940) mediante la metodología de historias conectadas. Se analizará la circulación trasnacional de saberes y sus formas de apropiación tomando aportes de la teoría de internacionalización Schriewer (1997). Las fuentes empleadas son boletines de asociaciones magisteriales y publicaciones particulares de maestros.

Palavras-clave: Circularción. Saberes. México. Uruguay. Maestros.

\section{Abstract}

The Uruguayan ruralist pedagogy and its connections with Mexican rural education (1930-1940) will be analysed through the methodology of connected stories. The transnational circulation of knowledge and its forms of appropriation will be analyzed, taking into consideration contributions from the Schriever internationalization theory (1997). The sources used are bulletins of teachers' associations and teachers' private publications. Keywords: Circulation. Knowledge. Mexico. Uruguay. Teachers. 


\section{Introducción}

Para quienes realizamos un estudio desde una perspectiva trasnacional y/o de historias conectadas es clave comenzar preguntándonos cómo circulaban los saberes; de qué modo se seleccionaban determinados contenidos como legitimadores para su uso fuera de los contextos donde fueron formulados; cómo fueron interpretados estos saberes en los diferentes contextos por donde circularon; $y$, más puntualmente en este trabajo nos preguntamos, qué experiencias sociales, culturales, educativas, propició su circulación su apropiación.

En este artículo nos ocuparemos de la pedagogía ruralista en Uruguay y sus conexiones con la educación rural mexicana entre las décadas 1930 y 1940 principalmente. Las fuentes a las que recurrimos para el mismo son publicaciones de asociaciones magisteriales tales como el Boletín U.N.M (Unión Nacional de Magisterio) y la Revista Concepto de la F.A.M.U (Federación Uruguaya de Magisterio), así como también otras publicaciones periodísticas escritas por maestros como Julio Castro. A su vez se tuvo en cuenta diversos documentos sin publicar del archivo del maestro Jesualdo Sosa, y referencias bibliográficas sobre el movimiento magisterial uruguayo, como el libro de Gonzales Sierra (1996) sobre la historia del gremialismo docente en Uruguay. Por último, un gran protagonista, cronista y posterior analista del movimiento pedagógico uruguayo entre 1940 y 1950, fue el recientemente fallecido maestro Miguel Soler Roca, a quien recurrimos en diversas ocasiones para conocer esta historia desde su perspectiva.

En el ámbito de la historia de la educación latinoamericana, las producciones académicas sobre pedagogías ruralistas tuvieron mayor desarrollo en México tras el impulso que recibió la misma luego de la revolución, esta constituyó una política de Estado implementada por los gobiernos federales posrevolucionarios (1920-1940). Fue una estrategia para ampliar su poder territorial en todo el país (Rockwell, 2018) y fomentar el desarrollo de las áreas rurales mediante políticas sociales y económicas, como lo fue la reforma agraria, las cuales implicaron, en muchos casos, una modernización de la vida campesina e indígena. Si bien tuvo un importante desarrollo en México y así lo reflejan las investigaciones al respecto ${ }^{1}$, la emergencia de este tipo de pedagogías se dio en distintos países de la región a partir de 1930. Con la crisis del capitalismo a nivel mundial y del sistema agro exportador, se intensificó el proceso de migración interna en distintos países de América Latina. Los avances tecnológicos, científicos y económicos se concentraron en las grandes ciudades, marcando más aún las desigualdades entre los espacios rurales y urbanos. En Uruguay (Santos, 2018) y Brasil (Werle \& Carvalho, 2009) durante las décadas de 1930 y 1940 se organizaron congresos y conferencias de educación en las cuales se debatió la despoblación de los campos y qué rol podía jugar en la escuela para frenar ese proceso. Estos debates también se replicaban a nivel internacional, en la Conferencia de Educación convocada por el Bureau International d'Educatión realizada en Génova 1938, se analizó el fenómeno migratorio a nivel mundial y se defendió la ruralización de la educación en los campos como una estrategia para enfrentarlo.

La resonancia trasnacional de esta preocupación y la elección, por parte de educadores involucrados en esta temática (con mayor o menor apoyo e intervención estatal en cada uno de los países), de las pedagogías ruralistas como una estrategia para intervenir en este escenario, da cuenta que las mismas no fueron un experimento educativo aislado o de carácter únicamente nacional. Su emergencia y desenvolvimiento entre 1930 y 1960 puede interpretarse como un proyecto de modernización que incidía en las relaciones entre las escuelas rurales y el desarrollo 
económico local, ampliando así los sentidos y prácticas de la educación escolar y del trabajo docente.

Ahora bien, dentro de un enfoque historiográfico trasnacional y de historias conectadas, la hipótesis que guía este trabajo es que la configuración de la pedagogía ruralista en Uruguay (1930-1960) tuvo lugar un proceso de internacionalización de experiencias y saberes pedagógicos ligados a la educación rural mexicana. Usamos los términos de configuración (Rockwell, 2007) y de internacionalización (Schriewer, 1997) porque consideramos que en Uruguay los maestros y las maestras involucrados en el movimiento pedagógico ruralista entrelazaron de un modo singular sus intereses, preocupaciones y proyectos (lo local), con aspectos que consideraban relevantes de experiencias de otros países, tomando como punto de referencia central a México. En este sentido, descartamos la hipótesis de que hubo un proceso de imposición de un país sobre otro o un trasplante de modelos. Por el contrario, resaltamos la capacidad de agencia de estos actores, los educadores y las educadoras (con sus iniciativas, intencionalidades, contradicciones y tensiones), en el devenir de la educación pública en su país y de la región.

La noción de configuración permite analizar estas conexiones trasnacionales y sus apropiaciones locales, ya que hace referencia a una lógica procesual mediante la cual una construcción social adquiere una forma específica. Esta lógica procesual se vincula con la noción de movimiento, en la cual los cambios y las permanencias pueden marchar en paralelo como entrecruzarse. El horizonte de la temporalidad es diacrónico y el análisis se centra en lo constituyente de cada proceso (Granja Castro, 1998). En este sentido, las conexiones que estos maestros y maestras establecieron con México, mediante las redes de comunicación y de intercambio de experiencias y saberes, así como también la difusión y re contextualización interna dentro el magisterio de las "noticias mexicanas", pueden volverse claves de análisis para indagar en el proceso de internacionalización que habría intervenido en la configuración de la pedagogía ruralista en Uruguay. Al respecto, Roldan Vera estudió el papel que jugaron las redes de comunicación en procesos de internacionalización de saberes y sostuvo que estas no deberían interpretarse solamente como

la vía para la transmisión del conocimiento sino también como formadoras del mismo (...) Centrar la atención en la complejidad y multidireccionalidad del proceso comunicativo puede permitirnos un alejamiento de nociones estáticas de producción y recepción de ideas, y acercarnos, en cambio, a la dimensión relacional de todo proceso de generación de conocimiento (Roldán Vera, 2011, pp. 336-337).

En cuanto a las redes de comunicación trasnacional, en nuestro caso, podemos marcar como un punto a partir del cual dar inicio a esta indagación, el registro de que en 1935 circuló información sobre el proceso educacional mexicano, mediante una publicación de la Secretaria de Educación Pública (SEP) de 1927 sobre las Misiones Culturales, en el Instituto Normal de Magisterio de Montevideo (Alonso \& Scagliola, 2012). Si bien en determinados círculos intelectuales uruguayos es probable que el interés por conocer qué sucedía en México posterior a la Revolución haya existido desde la década anterior ${ }^{2}$, en este trabajo mostraremos como a partir de 1935 estas ideas y experiencias comenzaron a circular con mayor frecuencia dentro del magisterio ${ }^{3}$.

Este mayor grado de circulación de noticias mexicanas en el magisterio uruguayo puede vincularse también con el cambio de gobierno en México; el 1ro de diciembre de 1934 asumió en México la presidencia Lázaro Cárdenas, y que, durante 
su mandato, la educación en contextos rurales que venía impulsándose desde el gobierno federal durante la década anterior, cobró mayor grado de desarrollo durante su mandato, mediante la llamada "educación socialista" (Civera, 2008), al promover también la reforma agraria en distintas zonas del país. En este sentido nos preguntamos: ¿por qué esta información llamó la atención de un sector del magisterio y los motivó a seguir indagando en este tema?; ¿hubo una necesidad de búsqueda, de este sector del magisterio vinculado con la educación en contextos rurales, de alternativas pedagógicas en experiencias extranjeras?; ¿por qué? En Schriewer y su teoría de la internacionalización podemos encontrar pistas para ir construyendo respuestas a estos interrogantes.

Según esta teoría, existen ciertos momentos históricos en que las referencias internas de un grupo o sociedad son consideradas inadecuadas para alcanzar cierto objetivo y, por lo tanto, se buscan fórmulas en referencias externas; estas referencias son posteriormente recontextualizadas (esto es adaptadas al contexto local) e internalizadas, hasta que todo resabio interno en ellas es borrado y se conviertan nuevamente en parte de la autoreferencialidad del grupo, sociedad o nación (Roldan Vera, 2011, p. 299).

¿De qué modos la propuesta de Schriewer puede contribuir en explicar estas búsquedas y adaptaciones protagonizadas por los maestros uruguayos? Si bien sostenemos que no hay teoría que calce de manera perfecta en ningún proceso histórico concreto, y no es la intención de este trabajo forzar un encuadre teórico, consideramos que hay aspectos planteados en la teoría de la internacionalización que pueden servir para interpretar y analizar cómo fue la constitución de la esta pedagogía en Uruguay. En este caso no hubo un proceso de internacionalización de un método educativo estructurado, como pudo haber sido la introducción del método de enseñanza mutua en Hispanoamérica en siglo XIX, proceso estudiado por Roldán Vera (2011), sino más bien conexiones y adaptaciones de ideas y experiencias pedagógicas vinculadas con la educación rural. Las prácticas, proyectos y políticas educativas mexicanas que tuvieron lugar entre 1920 y 1940 fueron múltiples, complejas y no exentas de conflictos y disputas de diversa índole y no constituyeron un método o modelo educativo definido ${ }^{4}$. Los maestros y maestras de Uruguay, interesados en la educación rural mexicana, no buscaban "fórmulas" para aplicar en su país; sostenían que las diferencias sustanciales que había entre ambas naciones, diferencias demográficas, culturales, en los procesos políticos, etc., harían imposible dicho trasplante. En todo caso, se podría plantear que buscaban inspiraciones, aportes, ideas para repensar la educación en los contextos rurales de su país. Vale aclarar aquí que en Uruguay durante en la década de 1930, la discusión sobre cómo debía ser la educación en los campos por parte maestros, inspectores y demás autoridades educativas, comenzó a polarizarse entre aquellos que estaban a favor la escuela rural tenga una orientación específica y quienes estaban en contra de esta diferenciación, siendo Castro uno de los impulsores de esta reforma ruralista. En sentido, las noticias sobre la educación rural mexicana encontraban cierto eco en los debates pedagógicos nacionales.

Ahora bien, siguiendo con la teoría de Schriewer, nos preguntamos, ¿Estas búsquedas y apropiaciones selectivas que efectuaron los maestros uruguayos pueden interpretarse como "externalizaciones"? Las externalizaciones, según el autor, son aquellas operaciones que vuelven accesible, de una forma filtrada, el ambiente internacional de un sistema y sus antecedentes históricos, de acuerdo a las cambiantes necesidades internas de "significado suplementario". Estas últimas, al variar no sólo entre naciones sino también de un periodo histórico y social a otro, 
implican construcciones, deconstrucciones y reconstrucciones de lo internacional, que se dan justamente, mediante las externalizaciones. Una vez que, para determinado grupo social ${ }^{5}$, el ambiente internacional se ha vuelto accesible,

\begin{abstract}
el concepto de externalización exige prestar una atención particular, dentro de los discursos educativos de las diferentes naciones o sociedades, a la recepción selectiva, la transformación interpretativa y las distintas modalidades de apropiación de los cuerpos de conocimiento, los modelos pedagógicos y las políticas educativas que se han figurado a nivel internacional (Schriewer, 2013, p. 284).
\end{abstract}

Analizando nuestro caso de estudio, consideramos que por parte del movimiento magisterial uruguayo hubo un proceso de búsqueda intencional de información sobre el proceso educacional y político mexicano y que estas búsquedas no deberían entenderse únicamente como una "recepción selectiva". Esta fue información que ellos procuraron activamente, y que, en algunos casos, hasta viajaron a México para interiorizarse del tema ${ }^{6}$, para luego poner sus interpretaciones en debate dentro del magisterio uruguayo. Frente a esa búsqueda de información (saberes, experiencias, proyectos, etc.), sí hubo una "recepción selectiva, transformaciones interpretativas y distintas modalidades de apropiación" (Schriewer, 2013:284) entre el movimiento magisterial, durante en un periodo extenso que abarcó prácticamente tres décadas (1930-1960).

A continuación, nos dedicaremos a fundamentar las observaciones que adelantamos en esta introducción.

\title{
Noticias mexicanas en el magisterio uruguayo
}

El proceso de organización política, social y cultural posterior a la Revolución Mexicana generó mucha curiosidad en la región. Particularmente, para quienes estaban vinculados con la ruralidad y la educación, noticias sobre el avance de la reforma agraria y la creación de escuelas rurales en América Latina, generaban un especial interés. Focalizándose aquí en la etapa que hemos denominado de invención de la pedagogía ruralista uruguaya $(1933-1942)^{7}$, podemos identificar circulación de información sobre la educación rural mexicana entre el magisterio uruguayo a partir de la década de 1930. Como mencionamos anteriormente, en 1935 llegó a los Institutos Normales de Montevideo información sobre las Misiones Culturales por medio de una publicación de la Secretaria de Educación Pública (SEP) de 1927 (Alonso y Scagliola, 2012). Tres años después, en 1938, se desarrolló en el Ateneo de Montevideo la semana de la Cultura Mexicana y en ella se hizo "referencia nuevamente a las Misiones Culturales" (Alonso y Scagliola, 2012, p. 69).

Con la creación en 1937 del Boletín de la Unión Nacional de Magisterio (U.N.M), las novedades sobre la educación rural mexicana comenzaron a ser difundidas por este periódico. La circulación de información empezó a adquirir mayor sistematicidad y fue intencionalmente buscada y seleccionada por parte de la U.N.M. El maestro y periodista Julio Castro, quien las décadas siguientes se convirtió en un actor clave en las conexiones en materia educativa entre Uruguay y México ${ }^{8}$, fue el Secretario de Redacción del mismo durante 1937 y Redactor Responsable entre 1940 y $1941^{9}$. Su accionar allí contribuyó a que se mantenga vigente el debate pedagógico en torno la educación rural en su país, mediante la publicación de diversas notas en que se abordaba a la educación rural como un problema nacional, incluyendo textos escritos por maestros uruguayos y notas redactadas por el mismo Castro sobre el tema ${ }^{10}$. A 
su vez, en el Boletín, se publicaba con frecuencia noticias referidas a la educación rural mexicana. En el año 1937, se identificó cinco artículos en la columna titulada "Noticiario del exterior" (U.N.M, 1937) dedicados a difundir los avances de México en ese ámbito ${ }^{11}$. En este sentido, se puede ver como los debates locales en torno a la educación rural y las noticas mexicanas sobre la misma, aparecían de forma intercalada en las páginas del Boletín de la U.N.M, construyendo un complejo temático vinculado con problemas y proyectos en torno a la educación rural en ambos países.

El interés por la difusión y debate en torno a estos temas continuó el año siguiente. En el Boletín se hace referencia a "Las charlas de los sábados"; estas eran "reuniones de estudio" organizadas por la U.N.M. Allí se mencionaba que las "charlas" se habían realizado con "mucho éxito", contando con la participación de maestros, profesores y estudiantes, y se invitaba a los lectores participar de los próximos encuentros (UNM, 1938:8). La primera "Charla" la había brindado Castro sobre "el movimiento histórico mejicano que dio como origen la reforma escolar" (U.N.M, 1938, p. 8); la segunda, la ofreció otro maestro, Napoleón Pradines Brazil, quien disertó sobre "La escuela en Mejico"; en la tercera se ofreció un concierto de guitarra; y para la cuarta, se "escogió el siguiente tema: ¿hay aspectos de la reforma escolar mejicana que convenga hacer nuestros?" (U.N.M, 1938, p. 8). Esta pregunta puede interpretarse como un indicio de que el interés de un sector del magisterio por conocer qué sucedía en las escuelas rurales mexicanas no era una curiosidad epistemológica y/o ideológica, sino que estaba motivada por el interrogante sobre la conveniencia de la apropiación, "hacer nuestros", de algunos aspectos de la reforma escolar mexicana. A su vez, esta intención de "hacer nuestros" puede estar indicando un proceso de "internalización" de referencias externas (Roldán Vera, 2011).

Por fuera del Boletín en 1938 Julio Castro publicó un documento periodístico titulado "Síntesis del Proceso Educacional Mexicano". En dicho texto, analizó en detalle distintos aspectos del proceso educacional mexicano y se preguntó, tal como se formuló en la cuarta Charla, qué pueden aportar estas experiencias educativas para repensar la educación rural en su país. Según Castro, el movimiento ligado a la educación rural en México, era el más "interesante" de su época y, el estudio estas realizaciones educacionales no podía hacerse de forma escindida de un análisis de la "realidad social, étnica, económica e histórica del país"; tarea a la cual se dedicó en este documento y en el que fue introduciendo comparaciones, reflexiones, críticas y propuestas para la educación rural en su país. La lectura su texto permite afirmar que mirar a México para Castro, era una forma para repensar su país y la educación en él (Autora, 2019). En un apartado de esta "Síntesis...", titulado "Hacia la ruralización", el autor señaló que "México va así a la vanguardia de un movimiento que se inicia en estos momentos en materia de educación, orientado a reivindicar la especificidad, diríamos, de la escuela rural" (1938, p. 24). Explicó que el fenómeno de "creciente urbanismo y la inquietante despoblación de la campaña" era mundial y que además de las causas económicas que lo generó, la huida del campo era "consecuencia, en no pequeña escala, del régimen educacional que ha orientado la enseñanza pública en todo el mundo" (1938, p. 24), un régimen educativo no ruralista, pensando para los habitantes de las ciudades. En esta defensa de la ruralización de la educación, el maestro apeló no sólo a México como "vanguardia" de un movimiento internacional, sino también mencionó la 5ta Conferencia Internacional Bureau International d'Educatión, la cual estuvo dedicada a estudiar el fenómeno de despoblación de los campos, donde los 43 países participantes firmaron un documento en favor por ruralización de la educación como una posible solución a este problema. Y, por último, se refirió además a la existencia de Escuelas Normales Rurales en otros países. 
Las tres menciones anteriores pueden interpretarse como una búsqueda de legitimación en el escenario internacional de la tendencia ruralista por parte de Castro. Siguiendo a Schriewer (1997) podemos plantear que Castro empleó un "discurso reformador internacionalizador" como una estrategia de legitimación mediante la externalización. Esta operación discursiva tiene su lógica ya que "demostrar la internacionalidad de las propias exigencias de reforma ha significado defenderlas contra el reproche de que son condicionadas y parciales, al mismo tiempo que ha significado cualificarlas como universales e indispensables" (Zymek, 1975, p. 348 en Schriewer, 1997, p. 46). De esta forma, la externalización de las situaciones mundiales se vuelve útil para justificar las reformas internas que Castro quería impulsar en su país.

Otra etapa comenzó a vislumbrarse en el escenario nacional cuando, en 1942, se celebraron elecciones nacionales libres y ganó la fórmula del "candidato de la democracia", el Doctor Juan José Amézaga. Finalizada la Segunda Guerra Mundial, Uruguay se alió con EEUU y producto a una reactivación económica, impulsó diversas obras públicas en el país y con ello logró el descenso del nivel de desocupación. En su gobierno se crearon las leyes de Consejos de Salarios, licencia anual, indemnización por despido e igualdad de los derechos de la mujer (Nahum, 2011). En este nuevo contexto político, las denuncias públicas que hicieron los maestros sobre las malas condiciones de vida en que estaban distintas poblaciones rurales, comenzaron a ser escuchadas por parte del Estado y tomaron en cuenta distintas propuestas pedagógicas que los maestros estaban elaborando.

Esta nueva forma de vinculación con el Estado por parte de este movimiento magisterial, marca, a los fines analíticos, el inicio a la segunda etapa en este proceso de configuración de la pedagogía ruralista en Uruguay que hemos denominado "etapa instituyente" (1943-1949) $)^{12}$. Durante estos años, los maestros y maestras se organizaron mediante congresos y pudieron desarrollar diversos proyectos pedagógicos como las Escuelas Granja, las Misiones Socio Culturales y el Instituto Normal Rural, entre otros. Un espacio de encuentro importante fue el Congreso Nacional de Maestros sobre Escuela Rural impulsado por, la recientemente creada, Federación Uruguaya de Magisterio (FAMU). Su primera parte fue 1944 y la segunda en 1945. El temario de la primera parte era amplio y diverso ${ }^{13}$, sin embargo, los maestros y las maestras rurales que allí se encontraron parecen haber instalado un problema como trasversal en todas las comisiones, el del latifundio y la consecuente necesidad de una reforma agraria ${ }^{14}$. Al respecto, Soler Roca, uno de los maestros allí presentes, destacó la siguiente conclusión arribada en el congreso: "El lamentable estado económico y social del niño se deriva de la irracional y regresiva estructura económica del medio" (Soler Roca, 1987, p. 41). Los maestros y maestras allí reunidos vinculaban esta estructura económica del medio con la finalidad que para ellos tenía la escuela en dicho entorno, y por ello sostenían que "la acción de la escuela no adquirirá su verdadera significación ni alcanzará las proyecciones ambicionadas por los maestros mientras no se realice la Reforma Agraria que transforme nuestro actual régimen económico" (Soler Roca, 1987, p. 41).

La demanda del congreso de maestros era explícita y su destinatario era claro: el gobierno nacional. Este se hizo presente para oír las voces de los maestros organizados. El Presidente, el Dr. Juan José de Amézaga, junto con el Ministro de Instrucción Pública, Dr. Adolfo Folle Juanicó y las autoridades de la enseñanza primaria y técnica, asistieron la sesión inaugural del Congreso celebrado en 1944, del mismo modo que habían estado presente en el Congreso Nacional de Maestros celebrado el año anterior. Su presencia allí puede ser interpretada como una señal de compromiso del gobierno con el magisterio y de expectativa de la FAMU para con el 
gobierno. Al respecto, en la editorial de la Revista Concepto ${ }^{15}$ publicada luego del congreso, se manifestaba lo siguiente:

Trasmitimos a nuestros hombres de gobierno estos puntos de esencial importancia, pedidos en el Congreso: reforma agraria, creación de fuentes de trabajo, construcción de caminos, aumento de sueldo a los maestros, progresiva protección a los escolares, atención a la opinión técnica de los maestros (Balbi, 1944, p. 1).

El debate entorno de la reforma agraria no era una novedad en Uruguay, durante la primera mitad del siglo $X X$ se presentaron varios proyectos legislativos de reforma agraria sin que ninguno tenga su correlato en la práctica. Al respecto, Soler Roca consideró que, en su país, "la reflexión sobre la distribución de la tierra era mucho más intelectual que reivindicativa" (Soler Roca, 1996, pp. 391-392). Esto se puede deber a distintos factores, en primer lugar, en términos demográficos, la mayor parte de la población ya residía en centros urbanos. A su vez, según los maestros rurales, muchos de los pobladores rurales vivían aislados unos de otros, existiendo en varias regiones una distancia de $5 \mathrm{~km}$ entre casa y casa; este "aislamiento" geográfico dificultaba los procesos de organización comunitaria y no contaban con otro centro social que no sea la escuela. Otros, sobre todo el norte del país, vivían en condiciones de hacinamiento en "rancheríos" que bordeaban grandes estancias ganaderas (Ferreiro, 1937). En este sentido, esta particular distribución demográfica puede haber dificultado la constitución de un movimiento de "campesinos"16, como sí sucedió en otros países latinoamericanos ${ }^{17}$. En Uruguay eran otros sectores, que no dependían directamente de la producción rural, como los maestros y las maestras rurales, quienes promovían estas reformas junto con cierto sector crítico de la intelectualidad de Montevideo.

Si bien esta demanda estaba en agenda para cierto sector de la sociedad uruguaya, nos preguntamos ¿Qué trascendencia pudo haber tenido el proceso de reforma agraria que había tenido lugar en México, particularmente durante el gobierno de Cárdenas (1934-1940), y su política educativa vinculada con la educación rural, en que se expresen tan claramente estas demandas en favor de la reforma agraria en el Congreso de Maestros sobre Escuela Rural en Montevideo (1944-145)?

Para responder esta pregunta contamos con algunos datos. En primer lugar, en la etapa de invención de la pedagogía ruralista uruguaya analizada anteriormente, dimos cuenta de cómo circulaba dentro del magisterio información vinculada a las políticas educativas desarrolladas durante el gobierno de Cárdenas en México. Desde 1935, año en que en comenzó a circular información sobre las Misiones Culturales mexicanas en los Institutos Normales de Magisterio de Montevideo hasta el Congreso celebrado 1944, pasaron prácticamente diez años durante los cuales, maestros y maestras organizadas en espacios gremiales y estudiantes de magisterio, estuvieron leyendo y debatiendo sobre estas cuestiones.

En segundo lugar, en 1943 regresó a Montevideo el maestro Jesualdo Sosa, reconocido regionalmente por su libro "Vida de un maestro" (1935), el cual es un registro novelado de su trabajo en la Escuela Rural, n. 54 de Colonia del Riachuelo. Por las repercusiones que causó la publicación de su libro, el gobierno de Terra le impidió seguir ejerciendo su cargo de maestro, pero su reconocimiento le valió la invitación del gobierno mexicano para trabajar como asesor en la SEP, cargo que desempeño entre 1939 y 1942. En 1943, el gobierno uruguayo de Amézaga lo contrató para que trabaje como asesor educativo en el país y le retiró las sanciones que le había impuesto el gobierno de Terra en 1935. 
Su regreso a Montevideo no pasó desapercibo para el magisterio, se lo invito al Congreso Nacional de Maestros de 1943, se lo nombró director de la Revista Concepto de la F.A.M.U y, en el primer tomo de dicha revista el maestro Otto Nieman publicó un extenso artículo sobre el último libro de Jesualdo, "Problemas de la educación y la cultura en América", el cual había escrito en México, a pedido del Comité de Unidad del Magisterio de América (CUMA). En dicho artículo Nieman sostuvo que Jesualdo estaba en condiciones de elaborar este libro por su experiencia de trabajo en Uruguay y en México y por su conocimiento de la educación en otros países de la región (Nieman, 1943, pp.10-11).

A su vez, en el Congreso Nacional de Maestros sobre Escuela Rural (1944), Jesualdo presentó un trabajo titulado "La escuela rural en América, el caso particular de la mexicana y la experiencia que nos proporcionó" (Gonzáles Sierra, 1996, p. 67). El título de esta ponencia nos remite al nombre de la Charla de los Sábados que había organizado la U.N.M en 1938, en donde se preguntaba "¿Hay aspectos de la reforma escolar mejicana que convenga hacer nuestros?". Las expresiones "hacer nuestros" y "experiencia que nos proporcionó" está dando cuenta de un proceso de recontextualización y apropiación, siempre selectiva e intencional, de algunos aspectos de estas experiencias educativas mexicanas. Además de su participación en entornos gremiales, Jesualdo tras su regreso de México, escribió textos sobre la realidad mexicana y su visión sobre la educación en dicho país para presentarse en conferencias, y hasta un libro sobre la escuela rural mexicana sin publicar ${ }^{18}$. Estos documentos resultan valiosos ya que no se ha estudiado en profundidad qué hizo Jesualdo en México como tampoco, qué generó en Uruguay su regreso de dicho país.

En tercer y último lugar, cinco meses después de que se celebrara la segunda parte del Congreso Nacional de Maestros sobre Escuela Rural en febrero de 1945, en julio de ese mismo año, Julio Castro, quien era en ese entonces profesor en Institutos Normales de Montevideo, impulsó y acompañó a los estudiantes magisteriales en el desarrollo de la primera misión socio pedagógica en un rancherío ubicado en Caraguatá. La cercanía entre el congreso y la primera misión socio pedagógica desarrollada en Uruguay, puede ser analizada como un indicador de que el proyecto de llevar adelante misiones campo adentro estaba ya en el magisterio uruguayo ${ }^{19}$.

En síntesis, para responder pregunta sobre qué trascendencia pudo haber tenido el proceso de reforma agraria en México, particularmente durante el gobierno de Cárdenas (1934-1940), y su política educativa vinculada con la educación rural, en que se expresen tan claramente estas demandas en favor de la reforma agraria en el Congreso de Maestros sobre Escuela Rural en Montevideo (1944-1945) y, teniendo en cuenta los tres datos planteados anteriormente, podemos afirmar que hay indicios para suponer que las informaciones que los maestros y maestras manejaban sobre el proceso político y educacional mexicano favoreció de algún modo su lucha en torno a una distribución de la tierra más equitativa en su país.

\section{Reflexiones finales y preguntas para seguir indagando}

A modo de reflexión final, consideramos que este análisis histórico nos permite observar las formas singulares en que se entretejió lo pedagógico y lo político en el movimiento magisterial uruguayo entre las décadas de 1930 y 1940 . Los maestros y las maestras se posicionaron como sujetos políticos que demandaban transformaciones sociales y económicas, sustentadas, en parte, por un "discurso reformador internacionalizador" (Schriewer, 1997). Las reformas sociales que exigían, excedían a los reclamos sindicales y educativos clásicos, pero a su vez, eran la puerta 
para volver viables los proyectos y prácticas pedagógicas que anhelaban. Sabían que en México la reforma agraria acompañó el proceso de ruralización de la educación durante, principalmente, la década 1930, pero en Uruguay, el contexto político era muy distinto. Los maestros y maestras tuvieron que esperar hasta el retorno de la democracia en 1943 para demandar al gobierno reformas en la distribución de la tierra, al mismo tiempo que impulsaban proyectos pedagógicos ruralistas que promovían otras formas de vida, trabajo y producción rural.

Para los educadores uruguayos, asumir el trabajo docente desde una perspectiva social, visión sobre la tarea pedagógica asociada posiblemente a las referencias que tenían sobre los maestros rurales mexicanos, puede haberlos interpelado y sensibilizado en relación a las condiciones de vida en que se encontraban distintas poblaciones rurales en su país. Y por ese motivo, su lucha como trabajadores de la educación, se volvió también una lucha política, que, de algún modo, fue inspirada por las noticias que tenían del proceso político, social y educativo de México en su periodo posrevolucionario.

Queda pendiente para un próximo trabajo analizar qué sucedió en cuanto al proceso de internacionalización durante la década de $1950^{20}$. Para ese entonces, tanto el escenario internacional como el nacional (en México y en Uruguay), se habían transformado sustancialmente en relación a cuando comenzó este proceso en 1930. Posterior a la Segunda Guerra Mundial, se conformaron organismos internacionales, como la UNESCO y el CREFAL (con sede en México), que comenzaron a incidir en los proyectos educativos de las naciones del "tercer mundo". En México, el periodo posrevolucionario (1920-1940), dejó ser un proceso vigente para pasar verse como una experiencia histórica a repensar, revisar y revalorizar, lo cual en parte se realizó desde el CREFAL. Y, en Uruguay, el movimiento instituyente del magisterio en pos de la educación rural, comenzó a ingresar en un proceso de institucionalización, en el cual organismos estatales cobraron cada vez más relevancia en la definición del rumbo de estas experiencias. En este marco, las vinculaciones de Uruguay con México continuaron, pero del proceso de externalización transitó hacia una internalización, en la cual, las referencias al periodo pos revolucionario mexicano se vuelven menos frecuentes, y a su vez, quedan cada vez más lejos en el tiempo y adquieren nuevas significaciones en el marco de un escenario internacional y nacional diferente. Para continuar con esta indagación será necesario recuperar lo planteando por Granja Castro (1998) sobre analizar el proceso de configuración a partir de tomar al horizonte de la temporalidad como diacrónico para advertir lo constituyente de cada proceso.

\section{Referencias}

Alonso, M. \& Gabriel S. (coords.) (2012). Misiones sociopedagógicas del Uruguay (1945-1971), Documentos para la memoria. Consejo de Formación en Educación (ANEP). Montevideo.

Calderón Mólgora, M. A. (2018). México: de la educación indígena a la educación rural. En Revista Historia y Memoria de la Educación, 7, 153-190.

Civera, A. (2008). La escuela como opción de vida: la formación de maestros normalistas rurales en México 1921-1945. El Colegio Mexiquense. México. 
Civera, A. \& Costa Rico, A. (2018). Desde la historia de la educación: educación y mundo. En Revista Historia y Memoria de la Educación, Facultad de Educación, Islas Canarias 7, 9-45.

Ferreiro, A. (1937) La enseñanza primaria en el medio rural. Florensa y Lafon, Montevideo.

Gonzales Sierra, Y. (1996) Un siglo de acción gremial y pedagógica del magisterio. 50 años de lucha de la FUM.CIEDUR - FESUR, Mimeo, Montevideo.

Granja Castro, J. (1998). Formaciones conceptuales en educación. México: Departamento de Investigaciones Educativas-CINVESTAV y Universidad Iberoamericana.

Loyo, E. (1999). Gobiernos revolucionarios y educación popular en México, 19111928. El Colegio de México. México.

Moraga Valle, F. (2019). Educación, exilio y diplomacia: Vasconcelos, Mistral, Torres Bodet y la proyección internacional de sus ideas educativas, 1921-1964. En Revista de Historia de América, 61-94.

Nahum, B. \& otros (2011). Historia Uruguaya: Crisis política y recuperación económica, 1930-1958. Ed. de la Banda Oriental. Montevideo.

Palacios, G. (1999). La pluma y el arado. Los intelectuales pedagogos y la construcción sociocultural del "problema campesino" en México, 1932-1934. El Colegio de México. México.

Autora. (2019). Pedagogías ruralistas: con los pies en Uruguay y los ojos puestos en México (1920-1960). En Revista Mexicana de Historia de la Educación, 7(14). Doi https://doi.org/10.29351/rmhe.v7i14.195

Autora. (2020). Maestros cruzando fronteras, experiencias y saberes entre Uruguay y México (1930-1960). En SAHE - UNIPE Circulaciones, tránsitos y traducciones en la historia de la educación (2021) Buenos Aires. (En prensa).

Rockwell, E. (2007). Hacer escuela, hacer Estado: la educación posrevolucionaria vista desde Tlaxcala, El Colegio de Michoacán-ciesas, México

Rockwell, E. [2005] Bardas, cercos y llaves: El encierro de escuelas indígenas rurales. En Arata, Escalante y Padawer (2018) Elsie Rockwell: Vivir entre escuelas: Relatos y presencias ( pp. 355-390). CLACSO. Argentina.

Roldán Vera, E. (2011) Internacionalización pedagógica y comunicación en perspectiva histórica: la introducción del método de enseñanza mutua en Hispanoamérica independiente. En Caruso, M.; Tenorth, H.-E. (comp.) Internacionalización. Políticas educativas y reflexión pedagógica en un medio global (pp. 297-343). Buenos Aires. Granica.

Santos, L. (2018). El pensamiento pedagógico nacional a través del Movimiento de Educación Rural. En Revista Quehacer Educativo, Montevideo, documento (audiovisual) disponible

en:

https://www.youtube.com/watch?v=MV4UV0xClfA\&feature=youtu.be 
Schriewer, J. (1997). Sistema mundial y redes de interrelación: La internacionalización de la educación y el papel de la investigación comparada. En Revista Educación y Ciencia. 1(15), 21-28.

Schriewer, J. (2013) Cultura mundial y mundos de significado culturalmente específicos. En Educar em Revista, Universidad Federal de Paraná, 49, 275297.

Sosa, J. (1935) Vida de un maestro. Trilce. Montevideo.

Soler Roca, M. (1987). El movimiento a favor de una nueva escuela rural. En Angione A. M. et al. Dos décadas en la historia de la escuela uruguaya. El testimonio de los protagonistas. Edición de la Revista de la Educación del Pueblo. Montevideo

Soler Roca, M. (1996). Educación y vida rural en América Latina. Federación Uruguaya del Magisterio. Instituto del Tercer Mundo. Montevideo.

Vaughan, M. K. (2000). Política cultural en la Revolución mexicana: maestros, campesinos y escuelas en México, 1930-1940. Fondo de Cultura Económica, SEP. México.

Werle, F. y Carvalho, A. M. (2009). A educação para a zona rural no sul do Brasil: sentidos e perspectivas a partir das conferências brasileiras de educação. En González T. y López O. (coords.) Educación rural en Iberoamérica.

\section{Documentos de archivo}

Anales de Instrucción Primaria (1929). La acción de los maestros mejicanos. Dirección de Enseñanza Primaria y Normal (pp. 157-158) Tomo XXX, n. 1, Montevideo. Febrero de 1929.

Castro, Julio (1937). Comentarios de un excelente libro "La enseñanza primaria en el medio rural" por A. Ferreiro. Unión Nacional de Magisterio. U.N.M. Año I (7).

Castro, J. (1938). Síntesis del Proceso Educacional Mexicano. Oficina de Prensa de Periodistas Libres. Disponible en: http://www.autoresdeluruguay.uy/biblioteca/julio_castro/lib/exe/fetch.php?medi a=sintesis_del_proceso_educacional_mexicano.pdf. Fecha de consulta: 21 jun. 2021.

Di Giorgi, D. (1941). Fines de la escuela rural. Unión Nacional de Magisterio Año III.

Luis O. J. (1940). La asistencia escolar en el medio rural. Unión Nacional de Magisterio. U.N.M Año IV.

Nieman, O. (1943). Bibliografía pedagógica. Revista CONCEPTO, 5 (1), 10-11.

Unión Nacional de Magisterio. (1937). La cuestión de la educación rural. U.N.M. Año I(7). 
Unión Nacional de Magisterio. (1937). Adaptación al medio. U.N.M. Montevideo. Año I.

Unión Nacional de Magisterio. (1937). Hacia la escuela rural. U.N.M. Año 1(2).

Unión Nacional de Magisterio (1941). Sugestiones para el trabajo escolar. Año V.

Unión Nacional de Magisterio (1937). La Gran Obra Educacional De México En Los Tres Últimos Años Unión Nacional de Magisterio. Año I (7).

Unión Nacional de Magisterio (1937) El Congreso Nacional de Educación de México UNM. Año I (5).

Unión Nacional de Magisterio. (1937). Mejico Unión Nacional de Magisterio. Año I (5).

Unión Nacional de Magisterio (1938). El Progreso De La Escuela Mexicana. UNM. Año I(8).

Unión Nacional del Magisterio (1938). Charlas de los sábados. UNM. Año I(8).

Selmar, B. (1944). Editorial. El Congreso Nacional de Maestros. Revista CONCEPTO $5(1)$

\section{Notas}

\footnotetext{
${ }^{1}$ Las investigaciones sobre educación rural en el México posrevolucionario (1920-1940) son numerosas y cada una hace un aporte desde una perspectiva particular (cultural, política, local, regional, nacional). Como trabajos ya clásicos es necesario mencionar a Loyo (1999), Civera (2008), Vaughan (2000), Rockwell (2007), Palacios (1999); mientras que en los últimos años destacan las investigaciones de Moraga (2019) y Calderón (2018) sobre este periodo, el primero hace referencia sobre todo a la figura de Vasconcelos y sus sucesores y el segundo problematiza el pasaje de la "educación indígena" a la "rural".

${ }^{2}$ En Autoria (2019) planteo como hipótesis que la vinculación del abogado y periodista uruguayo Carlos Quijano con políticos e intelectuales mexicanos, que había entablado con su visita a dicho país en 1925 tras ser invitado por el gobierno de Calles, puede haber propiciado la circulación de saberes sobre el México posrevolucionario en Uruguay. Esta invitación a México se produjo cuando Quijano estaba realizando estudios de posgrado en Francia y formaba parte de la agrupación AGELA con otros intelectuales latinoamericanos. La amistad entre Quijano y el maestro y periodista uruguayo Julio Castro, ambos fundadores en 1938 del reconocido Semanario Marcha, puede haber contribuido al interés del maestro por la educación rural en dicho país. Castro fue durante la segunda mitad de la década de 1930 uno de los principales difusores y analistas de lo que sucedía en torno a la educación rural mexicana en su país.

3 Previo a 1935, hasta el momento sólo se ha encontrado una referencia a la educación mexicana en una publicación de los Anales de Instrucción Primaria (1929) dentro de la sección "Información extranjera". La noticia se tituló "La acción de los maestros mejicanos" y hacía referencia la "lucha contra la superstición" (Anales de Instrucción Primaria, 1929, p. 157) que se estaba llevando adelante en México durante esos años. No se menciona nada vinculado a lo "rural". Esta nota fue a su vez extraída de la Revista Pour lere nouvelle, dirigida por el suizo Adolphe Ferrière, lo cual da cuenta de cómo circulaba la información la información entre México y Uruguay en ese periodo.

${ }^{4}$ Uno de los educadores uruguayos que más estudió y difundió el proceso educacional mexicano en su país fue Julio Castro. En un documento que publicó sobre dicho proceso en 1938, sobre "la actual escuela mexicana" expresó que "Es socialista en cuanto es una escuela para la sociedad, estructurada según las necesidades y los fines que de ella espera el movimiento social mexicano; ha sido realizada a la medida de las adaptaciones que tuvo que sufrir y de los obstáculos que tuvo que vencer" (Castro, 1938 , p. 4). Este análisis da la pauta de que los maestros uruguayos no escindían lo político y de lo
} 
pedagógico y que no encontraban en México un "modelo escolar" copiable, sino que lo analizaban como un proceso complejo y dinámico.

${ }^{5}$ Empleamos aquí el término grupo social porque en este caso estamos haciendo referencia a un sector del magisterio uruguayo vinculado con la educación rural y no a todo el país (Uruguay).

${ }^{6}$ Como los maestros Julio Castro, Jesualdo Sosa y Miguel Soler Roca, entre otros.

7 Esta denominación de esta etapa como "invención" forma parte del entramado conceptual de la tesis doctoral "La configuración de la pedagogía ruralista en Uruguay y sus conexiones con la educación rural mexicana" (Autora) que está en proceso de elaboración. Además de la etapa de invención (19331942) y la instituyente (1943-1949), en nuestra investigación abordamos la etapa de institucionalización (1950-1961), en la cual, las vinculaciones con México se mantienen y adquieren nuevas significaciones. Estas conceptualizaciones tienen como referencia a Castoriadis (1999). En este trabajo, solo destacaremos y analizaremos algunos aspectos de las dos primeras etapas.

${ }^{8}$ Castro denunció en diversas ocasiones las malas condiciones de vida en que se encontraban los habitantes de los rancheríos, escribió diversos libros y artículos sobre la educación en los campos, participó de actividades gremiales y de congresos de maestros, redactó programas educativos, y propició y acompañó el desarrollo de diversas experiencias pedagógicas en el ámbito rural. Entre 1946 y 1964, tuvo la oportunidad de viajar a México en diversas ocasiones, participar de congresos de educación rural, trabajar allí y brindar conferencias.

${ }^{9}$ Entre 1938 y 1940 Castro siguió formando parte de la U.N.M pero no hay registros de que haya tenido alguna función dentro del edición del Boletín. En 1939 fundó junto a Carlos Quijano el Semanario Marcha por lo que es posible que haya estado dedicado a dicha tarea.

10Publicaciones sobre educación rural en Uruguay en el Boletín de la U.N.M en 1937: "La cuestión de la educación rural”. U.N.M. Año I., 1937, p. 7; "Adaptación al medio" U.N.M. Montevideo. Año I., 1937; Castro (1937) "Comentarios de un excelente libro "La enseñanza primaria en el medio rural" por A. Ferreiro" U.N.M. Año I. 1937 p 7; "Hacia la escuela rural” Año 1,1937, p 2; Luis O. Jorge "La asistencia escolar en el medio rural" U.N.M Año IV., 1940; Di Giorgi. "Fines de la escuela rural" U.N.M Año III. 1941; "Sugestiones para el trabajo escolar" U.N.M. Año V .1941.

${ }^{11}$ En la sección "NOTICIARIO DEL EXTERIOR" del Boletín de la U.N.M, mientras Julio Castro era Secretario de Redacción se publicaron los siguientes artículos sobre México en 1937. "La Gran Obra Educacional De México En Los Tres Últimos Años Unión Nacional de Magisterio" U.N.M. Montevideo. Año I., 1937, p 7; "El Congreso Nacional de Educación de México Unión Nacional de Magisterio" U.N.M. Montevideo. Año I., 1937, p. 5; "Méjico" Unión Nacional de Magisterio U.N.M. Montevideo. Año I., 1937, p. 5; "El Progreso De La Escuela Mexicana Unión Nacional de Magisterio" U.N.M. Montevideo. Año I,. 1937 , p.5.

${ }^{12}$ Además de la etapa de invención (1933-1942) y la instituyente (1943-1949), en nuestra investigación abordamos la etapa de institucionalización (1950-1961), en la cual, las vinculaciones con México se mantienen y adquieren nuevas significaciones. Sin embargo, en este trabajo, solo destacaremos y analizaremos algunos aspectos de las dos primeras etapas.

13 "1er. tema: Estado actual de nuestra escuela rural: a) antecedentes del problema de la escuela rural; b) situación actual del medio rural; c) condiciones de la infancia en el medio rural; d) situación actual de la escuela rural en sus aspectos material, técnico y social; e) situación actual del maestro rural. 20. tema: Fines de la educación primaria en el medio rural: a) fines éticos, políticos y económico-sociales; b) fines artístico-afectivos; c) fines técnicos; d) fines de formación y educación física" (Soler Roca, 1987, p. 40).

${ }^{14} \mathrm{En}$ cuanto a las condiciones que se desarrollaba el trabajo pedagógico en algunos contextos rurales, Miguel Soler Roca reflexionó sobre su experiencia como maestro de una escuela rural unitaria en una zona de ganadería extensiva de Tacuarembó (1943-1945): "La inmensa riqueza pecuaria coexistía con la grave situación de miseria que afectaba a una reducida población que malvivía en los minifundios dispersos propios de los llamados rancheríos. De ese medio llegaban a mi escuela una veintena de niños pobres, algunos de ellos en situación de extrema pobreza" (Soler Roca, 2014, p. 135).

${ }^{15}$ Con la creación de la FAMU en 1944, se resolvió también fundar la revista "Concepto. Boletín pedagógico informativo de la FAMU", para la cual se designó como Director y Secretario de Redacción a Jesualdo Sosa.

${ }^{16}$ Empleo aquí el término "campesinos" en referencia al modo en que nombraban los maestros a los habitantes del campo uruguayo, en diferenciación de los "estancieros".

17 En el caso mexicano, "los regímenes posrevolucionarios (..) tuvieron que reconocer muchas de las demandas (incluidas por tierra y escuelas) de diversos grupos rurales que de hecho habían peleado para poner fin a la dictadura. Los regímenes sucesivos utilizaron ampliamente estas "conquistas revolucionarias", aunque en general solo retóricamente, para legitimar su dominio. Sin embargo, finalmente el proceso en sí de establecer un sistema escolar federal fortaleció el poder del gobierno centralizado unipartidario que perduró por el resto del siglo" (Rockwell, 2018, p. 386).

History of Education in Latin America - HistELA, v. 4, e25688, 2021, p. 14 de 15 
${ }^{18} \mathrm{El}$ archivo personal de Jesualdo Sosa se encuentra en la Biblioteca Nacional de Uruguay, en la sección de "Archivos literarios". De acuerdo a la documentación allí encontrada, Jesualdo presentó el 27 de abril de 1943 en el Rotary Club una conferencia titulada "Física, metafísica y actividad de México". También allí se encuentra un libro inédito del autor titulado "Mis experiencias pedagógicas en México", cuyo prólogo fue escrito en 1947. También, en dicho archivo, se encuentra otro texto sin publicar, titulado "Lo que los intelectuales y pueblos de lberoamérica esperan de la educación mexicana", sin fecha exacta pero escrito durante la década de 1940.

${ }^{19}$ Entre la primera parte del Congreso Nacional de Maestros sobre Escuela Rural (julio 1944) y la segunda parte (febrero 1945), se resolvió crear la FUM (Federación Uruguaya de Magisterio), que nucleaba a la F.A.M.U y a la Confederación de Maestros del Uruguay (C.M.U), unificando así las dos grandes organizaciones gremiales de maestros del país. Esta unificación "fue la síntesis de múltiples determinaciones, influencias y procesos. En su acto constitutivo resonaron los ecos de experiencias educativas y sociales conocidas y reflexionadas como las de México y Chile; los aprendizajes derivados de los acontecimientos de la República Española..." (Gonzáles Sierra, 1996, pp. 70-71). La F.U.M declaró, en su fundación, como meta inmediata en lo cultural, "Cruzadas culturales en el campo y la ciudad; Misiones pedagógicas (...). En lo que a la vida del pueblo se refiere: Lucha por la reforma agraria y otros problemas fundamentales del pueblo; Apoyo a las organizaciones de obreros y campesinos" (Gonzáles Sierra, 1996, p. 75)

${ }^{20}$ Para indagar en la pedagogía ruralista uruguaya durante la década 1950 y sus vinculaciones con México, se puede remitir a "Maestros cruzando fronteras, experiencias y saberes entre Uruguay y México (1930-1960)" (Autora, 2020). 